\title{
Observation of Magnetic Proximity Effect Using Resonant Optical Spectroscopy of an Electrically Tunable $\mathrm{MoSe}_{2} / \mathrm{CrBr}_{3}$ Heterostructure
}

\author{
Livio Ciorciaro $\odot,{ }^{1, *}$ Martin Kroner $\odot,{ }^{1}$ Kenji Watanabe $\odot,{ }^{2}$ Takashi Taniguchi, ${ }^{2}$ and Atac Imamoglu $\odot^{1}$ \\ ${ }^{1}$ Institut für Quantenelektronik, ETH Zürich, Auguste-Piccard-Hof 1, 8093 Zürich, Switzerland \\ ${ }^{2}$ National Institute for Materials Science, 1-1 Namiki, Tsukuba, Ibaraki 305-0044, Japan
}

(Received 6 February 2020; accepted 9 April 2020; published 14 May 2020)

\begin{abstract}
van der Waals heterostructures combining two-dimensional magnetic and semiconducting layers constitute a promising platform for interfacing magnetism, electronics, and optics. Here, we use resonant optical reflection spectroscopy to observe the magnetic proximity effect in a gate-tunable $\mathrm{MoSe}_{2} / \mathrm{CrBr}_{3}$ heterostructure. The high quality of the interface leads to a giant zero-field splitting of the $K$ and $K^{\prime}$ valley excitons in $\mathrm{MoSe}_{2}$, equivalent to an external magnetic field of $12 \mathrm{~T}$, with a weak but distinct electric field dependence that hints at potential for electrical control of magnetization. The magnetic proximity effect allows us to use resonant optical spectroscopy to fully characterize the $\mathrm{CrBr}_{3}$ magnet, determining the easy-axis coercive field, the magnetic anisotropy energy, and critical exponents associated with spin susceptibility and magnetization.
\end{abstract}

DOI: 10.1103/PhysRevLett.124.197401

Two-dimensional (2D) magnetic materials have attracted considerable attention due to their potential applications in spintronic devices $[1,2]$. Since the first demonstration that magnetism persists down to the monolayer limit in chromium trihalides $\left(\mathrm{Cr} X_{3}, X=\mathrm{Cl}, \mathrm{Br}, \mathrm{I}\right)[3,4]$, much progress has been made, both in understanding fundamental properties of these materials [5-14] and investigation of crucial steps towards applications [15-23]. Concurrently, transition metal dichalcogenides (TMDs) have established themselves as $2 \mathrm{D}$ semiconductors with remarkable optical properties [24-26] and possible applications in photonics and valleytronics [27-29]. van der Waals heterostructures composed of different 2D materials have the potential to realize atomically smooth interfaces that are not affected by lattice structure mismatch between the layers, allowing in principle arbitrary combinations of materials [30]. Magnetic proximity effect in such structures on the one hand leads to transfer of magnetization to otherwise nonmagnetic layers, and on the other hand may allow for controlling magnetization using electrical or optical excitation.

In this Letter, we use resonant optical spectroscopy to unequivocally demonstrate the magnetic proximity effect in a $\mathrm{MoSe}_{2} / \mathrm{CrBr}_{3}$ heterostructure, where we observe a large zero-field splitting of the $K$ and $K^{\prime}$ exciton resonances in $\mathrm{MoSe}_{2}$. We find that the magnetization of $\mathrm{MoSe}_{2}$ is exclusively induced by exchange coupling of conduction

Published by the American Physical Society under the terms of the Creative Commons Attribution 4.0 International license. Further distribution of this work must maintain attribution to the author(s) and the published article's title, journal citation, and DOI. band electrons. We use the shift of $\mathrm{MoSe}_{2}$ excitonic resonances to study the magnetic properties of $\mathrm{CrBr}_{3}$, and determine the magnetic anisotropy as well as the critical exponents associated with magnetization and susceptibility. Our work establishes resonant optical measurements in heterostructures incorporating TMD monolayers and 2D magnetic materials as a powerful spectroscopic tool that could be invaluable for studying magnetic materials with weak optical transitions without requiring high power laser excitation.

The sample we studied consists of a monolayer $\mathrm{MoSe}_{2}$ in direct contact with a bilayer $\mathrm{CrBr}_{3}$ encapsulated in hexagonal boron nitride $(h-\mathrm{BN})$ on a $\mathrm{SiO}_{2}$ substrate, as shown schematically in Fig. 1(a). In contrast to the layerantiferromagnets $\mathrm{CrI}_{3}$ and $\mathrm{CrCl}_{3}$, the interlayer exchange in bilayer $\mathrm{CrBr}_{3}$ has been shown to be ferromagnetic [4]. Monolayer graphene gates and a graphene contact allow for independent tuning of the charge carrier density and out-ofplane electric field in the sample. In the optical micrograph in Fig. 1(b), the regions of bare $\mathrm{MoSe}_{2}$, bare $\mathrm{CrBr}_{3}$, and the overlapping region can be seen. Details on the sample fabrication, optical setup, and data analysis are given in the Supplemental Material [31]. All measurements were performed at approximately $6 \mathrm{~K}$ unless stated otherwise.

Normalized polarization-resolved reflection spectra of the bare $\mathrm{MoSe}_{2}$ and the $\mathrm{MoSe}_{2} / \mathrm{CrBr}_{3}$ heterostructure are shown in Figs. 2(a) and 2(b), for a choice of gate voltages that ensure charge neutrality of $\mathrm{MoSe}_{2}$. In the absence of an external magnetic field, the $K$ and $K^{\prime}$ valley excitons are degenerate in bare $\mathrm{MoSe}_{2}$ and the spectrum shows no polarization dependence. In contrast, a valley splitting of $2.9 \mathrm{meV}$ emerges in the $\mathrm{MoSe}_{2} / \mathrm{CrBr}_{3}$ heterostructure region, equivalent to an external magnetic field of $12 \mathrm{~T}$, 


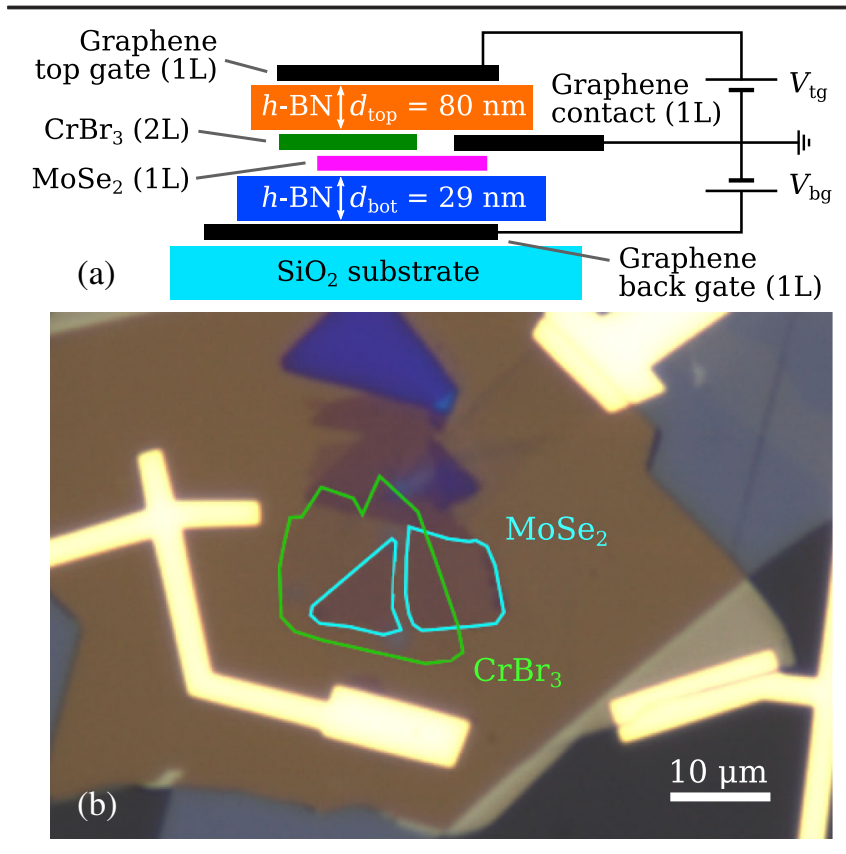

FIG. 1. (a) Schematic of the layer structure of the device and electrical connectivity. A bilayer $\mathrm{CrBr}_{3}$ and monolayer $\mathrm{MoSe}_{2}$ are encapsulated in $h$-BN. Monolayer graphene flakes are used as top and bottom gates and as contact to $\mathrm{MoSe}_{2}$. The stack is placed on a transparent $\mathrm{SiO}_{2}$ substrate. (b) Optical micrograph of the device, with $\mathrm{MoSe}_{2}$ and $\mathrm{CrBr}_{3}$ outlined in blue and green, respectively.

assuming the electronic $g$ factor to be 4 . This splitting can be attributed to an exchange coupling between electronic states in $\mathrm{MoSe}_{2}$ and spin-polarized states in $\mathrm{CrBr}_{3}$ that leads to different energy shifts for the $\mathrm{MoSe}_{2} K$ and $K^{\prime}$ valley excitons. Because of strain and disorder in the heterostructure, the splitting varies spatially by approximately $10 \%$.

To demonstrate that the $\mathrm{MoSe}_{2}$ exciton valley splitting originates from the magnetization of $\mathrm{CrBr}_{3}$, we compare hysteresis measurements of the $\mathrm{MoSe}_{2}$ reflectance as a function of an external out-of-plane magnetic field $B_{\perp}$ to the established method of measuring $\mathrm{CrBr}_{3}$ magnetization along the easy axis using the magneto-optical Kerr effect (MOKE) [4]. Figures 2(c) and 2(d) show the reflectance of a right- and left-hand circularly polarized $\left(\sigma^{+} / \sigma^{-}\right)$laser $(5 \mu \mathrm{W} \mathrm{CW})$ tuned to the low-frequency tail of the exciton resonance, as indicated by the vertical line in Fig. 2(b), as a function of $B_{\perp}$. For this choice of laser detuning, the valley splitting gives rise to maximal contrast in magnetic circular dichroism. The MOKE depicted in Fig. 2(e) is measured on the same spot with a linearly polarized laser at $2.755 \mathrm{eV}$ (450 nm, $20 \mu \mathrm{W} \mathrm{CW}$ ). The one-to-one correspondence between the measurements confirms that the valley splitting is directly linked to the magnetization of $\mathrm{CrBr}_{3}$. We verified that the MOKE signal is not altered by the presence of $\mathrm{MoSe}_{2}$ by comparing measurements on the heterostructure and bare $\mathrm{CrBr}_{3}$ (see Supplemental Material [31]). Using resonant spectroscopy on $\mathrm{MoSe}_{2}$ instead of the MOKE to access the magnetization of $\mathrm{CrBr}_{3}$ is advantageous, since it
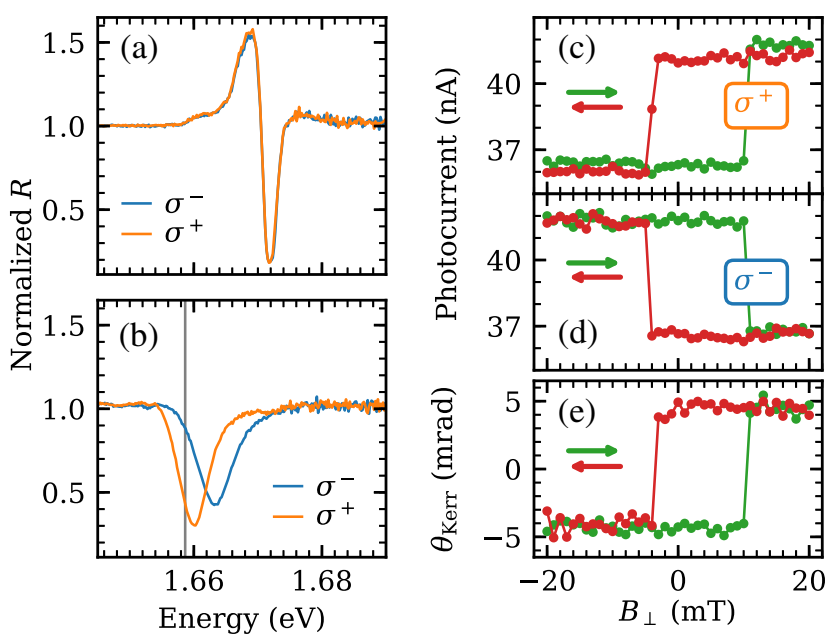

FIG. 2. (a) Reflection spectrum of bare undoped $\mathrm{MoSe}_{2}$. (b) Reflection spectra in $\sigma^{-}$and $\sigma^{+}$polarization of the undoped $\mathrm{MoSe}_{2} / \mathrm{CrBr}_{3}$ heterostructure at $B=0$. The $K$ and $K^{\prime}$ valley excitons are split by $2.9 \mathrm{meV}$. (c),(d) Hysteresis of the reflection of a $\sigma^{+} / \sigma^{-}$polarized laser tuned to the low-frequency tail of the $\mathrm{MoSe}_{2}$ exciton in the heterostructure as marked by the vertical line in panel (b). (e) Measurement of the $\mathrm{CrBr}_{3}$ magnetization hysteresis using the MOKE. The apparent offset from zero of the magnetic field in both measurements is due to a stray field from a ferromagnetic component in the cryostat.

allows us to perform the same measurement with a simpler technique and lower illumination power. Avoiding measurements requiring high laser intensity is particularly important for chromium trihalides where sizable MOKE signals are only obtained using above-band-gap lasers that could cause heating. Moreover, identifying peak positions instead of measuring laser intensities after a polarizing beam splitter makes our spectroscopic method less sensitive to imperfections in the polarization selection than traditional techniques.

An exchange splitting of similar magnitude has been reported in the pioneering work on photoluminescence (PL) measurements of $\mathrm{WSe}_{2} / \mathrm{CrI}_{3}$ heterostructures $[5,14,20]$. We were not able to observe the splitting in PL measurements (see Supplemental Material [31]). Instead, we see broad emission lines with an integrated intensity that is smaller by a factor of 20 compared to bare $\mathrm{MoSe}_{2}$, which suggests that tunneling to $\mathrm{CrBr}_{3}$ provides a fast nonradiative relaxation channel for conduction band electrons and excitons in $\mathrm{MoSe}_{2}$. Because the exchange coupling responsible for the splitting relies on second-order virtual tunnel coupling, the PL splitting is expected to be large where the tunnel coupling is large, leading to a short exciton lifetime. Since PL primarily originates from long-lived states, we would expect it to be dominated by low-oscillator-strength localized excitations in parts of the heterostructure where the tunnel coupling is small. Consequently, disorder-induced spatial variations of the tunnel coupling could lead to a PL signal that shows small or possibly vanishing exciton valley 

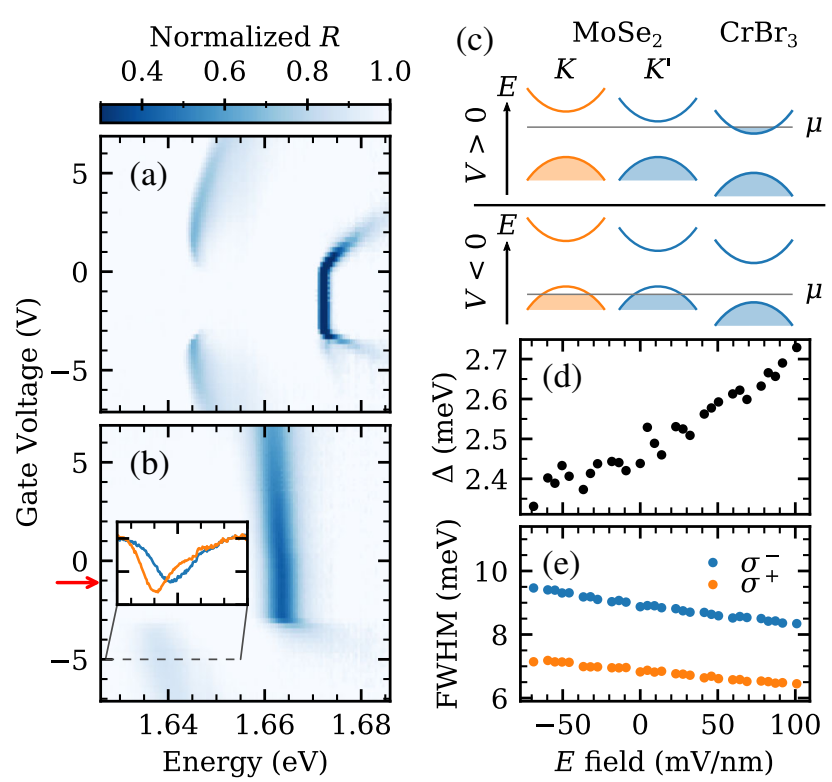

FIG. 3. (a) Gate dependence of the bare $\mathrm{MoSe}_{2}$ reflection spectrum. The appearance of attractive and repulsive polaron lines at positive and negative voltages shows the flake can be $n$ doped and $p$ doped. (b) Gate dependence of the reflection spectrum of the heterostructure in $\sigma^{-}$polarization. Because of the type-II band alignment, $\mathrm{MoSe}_{2}$ cannot be $n$ doped anymore, as signified by the persistence of the exciton line at positive voltages. Inset: Reflection spectra in the $p$-doped regime, as indicated by the dashed line. The attractive polaron lines show the same splitting as the exciton lines. The $x$ ticks are identical to the parent axis, the $y$ tick separation is 0.1. (c) Schematic of the type-II band alignment of $\mathrm{MoSe}_{2}$ and $\mathrm{CrBr}_{3}$ with chemical potential $\mu$ for positive and negative gate voltages. Exchange coupling leads to valley splitting in the conduction band of $\mathrm{MoSe}_{2}$. (d), (e) Dependence of the valley splitting $\Delta$ and FWHM of the exciton lines on the out-of-plane electric field. Both the splitting and FWHM are tunable, indicating a modification of the tunneling rate across the $\mathrm{MoSe}_{2} / \mathrm{CrBr}_{3}$ interface.

splitting. This is in contrast to resonant reflection and absorption measurements which probe extended states with high oscillator strength within the optical excitation spot.

To explore the nature of the exchange coupling between $\mathrm{CrBr}_{3}$ and $\mathrm{MoSe}_{2}$ we measure the gate voltage dependence of the reflection spectra in $\sigma^{-}$polarization, shown in Figs. 3(a) and 3(b). The voltages indicated on the vertical axis were applied to both the top and bottom gate. The bare $\mathrm{MoSe}_{2}$ flake can be charged with electrons or holes, evidenced by the appearance of attractive polaron lines at both positive and negative gate voltages [34]. In the presence of $\mathrm{CrBr}_{3}$, only holes can be injected into $\mathrm{MoSe}_{2}$, consistent with the type-II band alignment schematically shown in Fig. 3(c) and predicted from $a b$ initio calculations $[35,36]$. Injected electrons accumulate in the lower-lying conduction band of $\mathrm{CrBr}_{3}$, leaving the $\mathrm{MoSe}_{2}$ undoped and leading to screening of the top gate (see Supplemental Material [31]).

The attractive polaron line on the $p$-doped side exhibits the same valley splitting as the neutral exciton, as shown in the inset of Fig. 3(b). If the itinerant holes in $\mathrm{MoSe}_{2}$ were subject to a sizable exchange interaction, we would observe a strong valley polarization of holes, leading to a single circularly polarized attractive polaron resonance $[37,38]$. The observation of polaron resonances with equal strength for the two polarizations demonstrates that electron exchange is predominantly responsible for the exciton and polaron valley splitting. A detailed understanding of the underlying coupling mechanism is beyond the scope of this Letter and requires additional theoretical work.

The presence of top and bottom gates allows us to probe the electric field dependence of the reflectance for constant chemical potential. In the absence of mobile charges in the heterostructure, the electric field is approximately given by $E \approx\left(V_{\mathrm{tg}}-V_{\mathrm{bg}}\right) /\left(d_{\text {top }}+d_{\text {bot }}\right)$ and the chemical potential by $\mu \propto d_{\text {bot }} V_{\text {tg }}+d_{\text {top }} V_{\text {bg }}$, where $d_{\text {top }}$ and $d_{\text {bot }}$ are the thicknesses of the top and bottom $h$-BN flakes; the actual electric field may deviate by a constant factor due to the dielectric constants and finite thickness of $\mathrm{MoSe}_{2}$ and $\mathrm{CrBr}_{3}$ (see Supplemental Material [31]). To keep the chemical potential constant while varying the electric field, we tune the gate voltages with a fixed ratio $\left(V_{\mathrm{tg}}-V_{0}\right) / V_{\mathrm{bg}}=3.3$, where $V_{0}$ determines the chemical potential; we determined this ratio experimentally from 2D gate sweeps (see Supplemental Material [31]).

Figures 3(d) and 3(e) show the valley splitting and the reflection peak widths, respectively, for the neutral exciton as a function of the applied electric field. The choice of gate voltages, indicated by the red arrow in Fig. 3(b), ensures the charge neutrality of the heterostructure. Clear dependence of both the splitting and the linewidth on the electric field suggests that the tunnel coupling strength is modified. Such an approximately linear electric field dependence of the splitting was predicted in theoretical works $[35,36]$ and may have implications for future gate-tunable spintronic devices. Additionally, the higher-energy exciton line (here $\sigma^{-}$) is consistently broader than the lower-energy line, presumably due to the spin-dependent charge transfer between $\mathrm{MoSe}_{2}$ and $\mathrm{CrBr}_{3}$; similar observations were previously reported in heterostructures composed of different 2D magnetic layers [14,20].

Having demonstrated the magnetic proximity effect in $\mathrm{MoSe}_{2}$, we use resonant spectroscopy of the $\mathrm{MoSe}_{2}$ exciton resonance to determine the magnetic properties of $\mathrm{CrBr}_{3}$. Figure 4(a) shows fitted positions of the split exciton peaks as a function of an applied in-plane magnetic field $B_{\|}$. We observe that for $B_{\|} \geq 0.1 \mathrm{~T}$, the splitting gradually decreases and saturates at a value of $0.1 \mathrm{meV}$ for $0.3 \mathrm{~T}$. The reduction of the splitting is a consequence of the canting of the $\mathrm{CrBr}_{3}$ spins into the plane. The small remaining splitting at high magnetic fields is due to a tilt of the magnetic field axis with respect to the sample plane that leads to an out-of-plane component of the magnetization and consequently a nonzero exchange field. A striking feature of the data in Fig. 4(a) is the asymmetry in the 

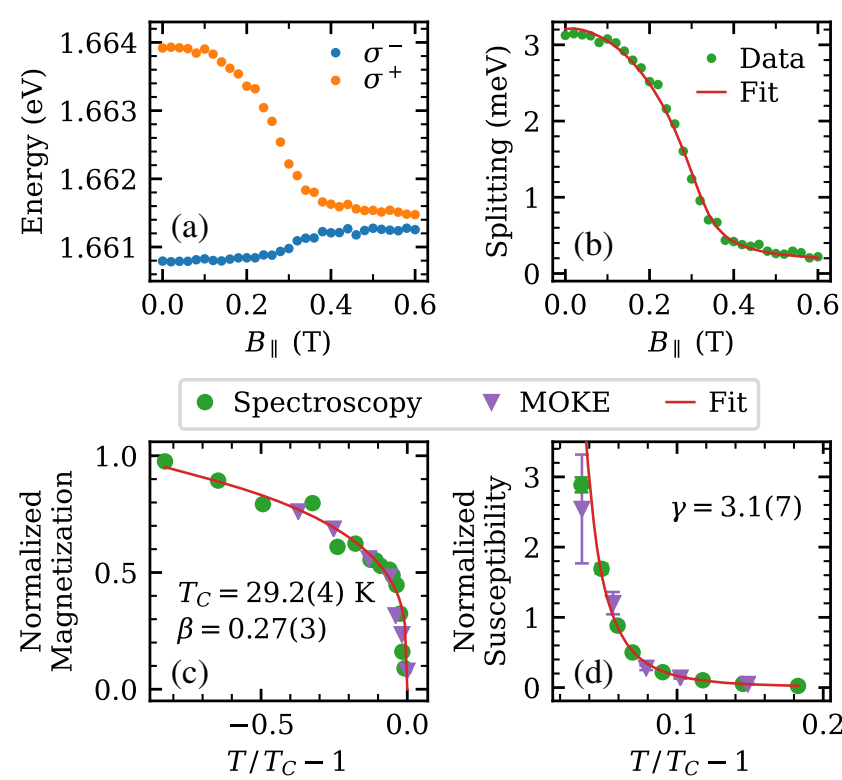

FIG. 4. (a) Fitted exciton peak positions from polarizationresolved reflection spectra as function of in-plane magnetic field. The splitting collapses around $0.3 \mathrm{~T}$, indicating the transition to inplane magnetization of $\mathrm{CrBr}_{3}$. (b) Exciton valley splitting as function of in-plane magnetic field. The fitted model using a macroscopic magnetic moment with anisotropy is in excellent agreement with the experimental data. (c) Magnetization of $\mathrm{CrBr}_{3}$ as a function of temperature. Round data points are extracted from polarization-resolved reflection spectra of $\mathrm{MoSe}_{2}$ and triangular data points are extracted from MOKE measurements. A fit of the form $m(T)=A\left(1-T / T_{C}\right)^{\beta}$ yields values for the critical temperature $T_{C}$ and critical exponent $\beta$. (d) Magnetic susceptibility as a function of temperature extracted from spectra and MOKE measurements. A fit of the form $\chi(T)=B\left(T / T_{C}-1\right)^{-\gamma}$ yields the critical exponent $\gamma$.

$B_{\|}$-induced change in resonance energy between the lowand high-energy exciton peaks: we speculate that this asymmetry could arise from an energy splitting between the spin-polarized conduction bands of $\mathrm{CrBr}_{3}$ that play a prominent role in determining the exchange coupling to $\mathrm{MoSe}_{2}$ electrons in different valleys.

The observed dependence of the splitting vs $B_{\|}$allows us to estimate the anisotropy energy of $\mathrm{CrBr}_{3}$. To this end, we assume that the $\mathrm{CrBr}_{3}$ flake has a uniform magnetization $\vec{m}$ and numerically minimize its potential energy $E=$ $K(\sin \theta)^{2}+|m| B_{\|} \cos (\theta-\alpha)$, where $K$ is the anisotropy energy along the easy axis, $\theta$ is the angle of the magnetic moment with respect to the easy axis, $B_{\|}$is the external magnetic field, and $\alpha$ is the angle of $B_{\|}$with respect to the easy axis. We set $|m|=3.87 \mu_{B}$ to the magnetization per $\mathrm{Cr}$ atom $[39,40]$ and fit the model to the experimental data to obtain an anisotropy energy $K=34.3(4) \mu \mathrm{eV}$ per $\mathrm{Cr}$ atom and $\alpha=88.20(13)^{\circ}$. Although this simple model does not tell us anything about the type of anisotropy, previous calculations have shown that it is expected to originate from anisotropic exchange coupling rather than on-site anisotropy [41]. By using the previously reported values of the isotropic intralayer exchange of bulk $\mathrm{CrBr}_{3}$ $(J \approx 0.8 \mathrm{meV})[42,43]$, we find that the exchange interaction is weakly anisotropic, $J / K \approx 20$. The small coercive field we measure [Figs. 2(c)-2(e)] is consistent with the weak anisotropy of the intralayer exchange interaction. Remarkably, the exciton valley splitting in $\mathrm{MoSe}_{2}$ is comparable to the $\mathrm{CrBr}_{3}$ intralayer exchange $J$, even though the exchange between two $\mathrm{CrBr}_{3}$ layers is expected to be significantly smaller [42-44].

Next, we measure the critical temperature $T_{C}$ and critical exponents of the second-order magnetic phase transition of $\mathrm{CrBr}_{3}$ through resonant $\mathrm{MoSe}_{2}$ exciton spectroscopy. To this end, we measure the hysteresis curves of circularly polarized reflection spectra vs $B_{\perp}$ as a function of temperature (see Supplemental Material [31]). For $T<T_{C}$, shown in Fig. 4(c), the splitting at $B_{\perp}=0$ provides a measure for the remnant magnetization $m$, which is the order parameter for the phase transition. For $T>T_{C}$, shown in Fig. 4(d), the slope of the splitting vs $B_{\perp}$ is proportional to the magnetic susceptibility $\chi$. By fitting the functional forms $m(T)=$ $A_{1}\left(1-T / T_{C}\right)^{\beta}$ and $\chi(T)=A_{2}\left(T / T_{C}-1\right)^{-\gamma}$ simultaneously to the experimental data, we find the Curie temperature $T_{C}=29.2(4) \mathrm{K}$ as well as the critical exponents $\beta=0.27(3)$ and $\gamma=3.1(7)$. We also perform similar measurements using the MOKE and find that the data points fall onto the same curves when normalized with respect to the peak values of $m$ and $\chi$. The values we obtain for $\beta$ and $\gamma$ are consistent with the 2D-Heisenberg model with weak anisotropy [45].

In conclusion, we use resonant exciton reflection measurements of valley splitting in $\mathrm{MoSe}_{2}$ to demonstrate a strong magnetic proximity effect due to ferromagnetic $\mathrm{CrBr}_{3}$. From the absence of itinerant hole valley polarization in the reflection spectra we infer that the resulting valley Zeeman effect is predominantly due to exchange coupling between conduction band electrons in $\mathrm{MoSe}_{2}$ and $\mathrm{CrBr}_{3}$ : remarkably, the strength of this interlayer exchange coupling is comparable to the intralayer exchange coupling in $\mathrm{CrBr}_{3}$. Our investigation of the magnetic properties of $\mathrm{CrBr}_{3}$ using resonant optical spectroscopy reveals several features such as an electric field dependence of the proximity effect, weak anisotropy $J / K \approx 20$ of the exchange interaction, and the critical exponents associated with the magnetic phase transition.

The data that support the findings of this Letter are available in the ETH Research Collection [46].

We gratefully acknowledge the contributions of Alberto Morpurgo and Zhe Wang who introduced us to the field of 2D magnets. Y. Shimazaki and A. Popert contributed to the fabrication of the heterostructure. We also express our gratitude to A. Vindigni and P. Maletinsky for insightful discussions. This work was supported by the Swiss National Science Foundation (SNSF) under 
Grant No. 200021-178909/1. K. W. and T. T. acknowledge support from the Elemental Strategy Initiative conducted by the MEXT, Japan, and the CREST (JPMJCR15F3), JST.

*livioc@phys.ethz.ch

[1] C. Gong and X. Zhang, Science 363, eaav4450 (2019).

[2] D. L. Cortie, G. L. Causer, K. C. Rule, H. Fritzsche, W. Kreuzpaintner, and F. Klose, Adv. Funct. Mater. 30, 1901414 (2019).

[3] B. Huang, G. Clark, E. Navarro-Moratalla, D. R. Klein, R. Cheng, K. L. Seyler, D. Zhong, E. Schmidgall, M. A. McGuire, D. H. Cobden, W. Yao, D. Xiao, P. JarilloHerrero, and X. Xu, Nature (London) 546, 270 (2017).

[4] Z. Zhang, J. Shang, C. Jiang, A. Rasmita, W. Gao, and T. Yu, Nano Lett. 19, 3138 (2019).

[5] D. Zhong, K. L. Seyler, X. Linpeng, R. Cheng, N. Sivadas, B. Huang, E. Schmidgall, T. Taniguchi, K. Watanabe, M. A. McGuire, W. Yao, D. Xiao, K.-M. C. Fu, and X. Xu, Sci. Adv. 3, e1603113 (2017).

[6] L. Chen, J.-H. Chung, B. Gao, T. Chen, M. B. Stone, A. I. Kolesnikov, Q. Huang, and P. Dai, Phys. Rev. X 8, 041028 (2018).

[7] W. Jin, H. H. Kim, Z. Ye, S. Li, P. Rezaie, F. Diaz, S. Siddiq, E. Wauer, B. Yang, C. Li, S. Tian, K. Sun, H. Lei, A. W. Tsen, L. Zhao, and R. He, Nat. Commun. 9, 5122 (2018).

[8] L. Thiel, Z. Wang, M. A. Tschudin, D. Rohner, I. GutiérrezLezama, N. Ubrig, M. Gibertini, E. Giannini, A. F. Morpurgo, and P. Maletinsky, Science 364, 973 (2019).

[9] C. Jin, Z. Tao, K. Kang, K. Watanabe, T. Taniguchi, K. F. Mak, and J. Shan, arXiv:1910.13023.

[10] T. Song, Z. Fei, M. Yankowitz, Z. Lin, Q. Jiang, K. Hwangbo, Q. Zhang, B. Sun, T. Taniguchi, K. Watanabe, M. A. McGuire, D. Graf, T. Cao, J.-H. Chu, D. H. Cobden, C. R. Dean, D. Xiao, and X. Xu, Nat. Mater. 18, 1298 (2019).

[11] X. Cai, T. Song, N. P. Wilson, G. Clark, M. He, X. Zhang, T. Taniguchi, K. Watanabe, W. Yao, D. Xiao, M. A. McGuire, D. H. Cobden, and X. Xu, Nano Lett. 19, 3993 (2019).

[12] W. Chen, Z. Sun, Z. Wang, L. Gu, X. Xu, S. Wu, and C. Gao, Science 366, 983 (2019).

[13] Z. Sun, Y. Yi, T. Song, G. Clark, B. Huang, Y. Shan, S. Wu, D. Huang, C. Gao, Z. Chen, M. McGuire, T. Cao, D. Xiao, W.-T. Liu, W. Yao, X. Xu, and S. Wu, Nature (London) 572, 497 (2019).

[14] D. Zhong, K. L. Seyler, X. Linpeng, N. P. Wilson, T. Taniguchi, K. Watanabe, M. A. McGuire, K.-M. C. Fu, D. Xiao, W. Yao, and X. Xu, Nat. Nanotechnol. 15, 187 (2020).

[15] Z. Wang, I. Gutiérrez-Lezama, N. Ubrig, M. Kroner, M. Gibertini, T. Taniguchi, K. Watanabe, A. Imamoğlu, E. Giannini, and A.F. Morpurgo, Nat. Commun. 9, 2516 (2018).

[16] T. Song, X. Cai, M. W. -Y. Tu, X. Zhang, B. Huang, N. P. Wilson, K. L. Seyler, L. Zhu, T. Taniguchi, K. Watanabe, M. A. McGuire, D. H. Cobden, D. Xiao, W. Yao, and X. Xu, Science 360, 1214 (2018).

[17] D. Ghazaryan, M. T. Greenaway, Z. Wang, V. H. GuarochicoMoreira, I. J. Vera-Marun, J. Yin, Y. Liao, S. V. Morozov, O.
Kristanovski, A. I. Lichtenstein, M. I. Katsnelson, F. Withers, A. Mishchenko, L. Eaves, A. K. Geim, K. S. Novoselov, and A. Misra, National electronics review 1, 344 (2018).

[18] D. R. Klein, D. MacNeill, J. L. Lado, D. Soriano, E. Navarro-Moratalla, K. Watanabe, T. Taniguchi, S. Manni, P. Canfield, J. Fernández-Rossier, and P. Jarillo-Herrero, Science 360, 1218 (2018).

[19] S. Jiang, L. Li, Z. Wang, K. F. Mak, and J. Shan, Nat. Nanotechnol. 13, 549 (2018).

[20] K. L. Seyler, D. Zhong, B. Huang, X. Linpeng, N. P. Wilson, T. Taniguchi, K. Watanabe, W. Yao, D. Xiao, M. A. McGuire, K.-M. C. Fu, and X. Xu, Nano Lett. 18, 3823 (2018).

[21] B. Huang, G. Clark, D. R. Klein, D. MacNeill, E. NavarroMoratalla, K. L. Seyler, N. Wilson, M. A. McGuire, D. H. Cobden, D. Xiao, W. Yao, P. Jarillo-Herrero, and X. Xu, Nat. Nanotechnol. 13, 544 (2018).

[22] S. Jiang, J. Shan, and K. F. Mak, Nat. Mater. 17, 406 (2018).

[23] M. U. Farooq and J. Hong, npj 2D Mater. Appl. 3, 3 (2019).

[24] X. Xu, W. Yao, D. Xiao, and T. F. Heinz, Nat. Phys. 10, 343 (2014).

[25] K. F. Mak, D. Xiao, and J. Shan, Nat. Photonics 12, 451 (2018).

[26] M. Onga, Y. Zhang, T. Ideue, and Y. Iwasa, Nat. Mater. 16, 1193 (2017).

[27] K. F. Mak, C. Lee, J. Hone, J. Shan, and T. F. Heinz, Phys. Rev. Lett. 105, 136805 (2010).

[28] S. Manzeli, D. Ovchinnikov, D. Pasquier, O. V. Yazyev, and A. Kis, Nat. Rev. Mater. 2, 17033 (2017).

[29] Y. Deng, Y. Yu, Y. Song, J. Zhang, N. Z. Wang, Z. Sun, Y. Yi, Y.Z. Wu, S. Wu, J. Zhu, J. Wang, X. H. Chen, and Y. Zhang, Nature (London) 563, 94 (2018).

[30] A. K. Geim and I. V. Grigorieva, Nature (London) 499, 419 (2013).

[31] See Supplemental Material at http://link.aps.org/ supplemental/10.1103/PhysRevLett.124.197401 for information on the sample fabrication, experimental details, data analysis, and extended data on gate dependence, photoluminescence, and optical doping, which include Refs. [32,33].

[32] F. J. Kopp and T. Ashworth, Rev. Sci. Instrum. 43, 327 (1972).

[33] L. Wang, I. Meric, P. Y. Huang, Q. Gao, Y. Gao, H. Tran, T. Taniguchi, K. Watanabe, L. M. Campos, D. A. Muller, J. Guo, P. Kim, J. Hone, K. L. Shepard, and C. R. Dean, Science 342, 614 (2013).

[34] M. Sidler, P. Back, O. Cotlet, A. Srivastava, T. Fink, M. Kroner, E. Demler, and A. Imamoglu, Nat. Phys. 13, 255 (2017).

[35] K. Zollner, P. E. Faria Junior, and J. Fabian, Phys. Rev. B 100, 085128 (2019).

[36] J. Xie, L. Jia, H. Shi, D. Yang, and M. Si, Jpn. J. Appl. Phys. 58, 010906 (2019).

[37] P. Back, M. Sidler, O. Cotlet, A. Srivastava, N. Takemura, M. Kroner, and A. Imamoglu, Phys. Rev. Lett. 118, 237404 (2017).

[38] T. Smoleński, O. Cotlet, A. Popert, P. Back, Y. Shimazaki, P. Knüppel, N. Dietler, T. Taniguchi, K. Watanabe, M. Kroner, and A. Imamoglu, Phys. Rev. Lett. 123, 097403 (2019). 
[39] M. Kotani, J. Phys. Soc. Jpn. 4, 293 (1949).

[40] J. F. Dillon, in Proceedings of the Seventh Conference on Magnetism and Magnetic Materials, edited by J. A. Osborn (Springer US, Boston, MA, 1962), pp. 1191-1192.

[41] J. L. Lado and J. Fernández-Rossier, 2D Mater. 4, 035002 (2017).
[42] H. L. Davis and A. Narath, Phys. Rev. 134, A433 (1964).

[43] E. J. Samuelsen, R. Silberglitt, G. Shirane, and J.P. Remeika, Phys. Rev. B 3, 157 (1971).

[44] L. de Jongh and A. Miedema, Adv. Phys. 23, 1 (1974).

[45] M. E. Fisher, Rev. Mod. Phys. 46, 597 (1974).

[46] See http://hdl.handle.net/20.500.11850/397581. 